\title{
IMPACTO DA ATIVIDADE FÍSICA SOBRE A MASSA ÓSSEA DE RATAS OSTEOPENICAS
}

Lorena F. Caixeta

Andre Adriano R. Aleixo

Gustavo S. Abrahão

Dayana P. P. de Siqueira

Leonardo C. Carvalho

Joao Paulo C. Matheus

Antonio C. Shimano

\section{Resumo}

Avaliamos a massa dos femures, de ratas ovariectomizadas na atividade física. Foram utilizadas 30 ratas divididas em grupos: G1: Controle. G2: Animais ovariectomizados e não submetidos a exercícios. G3: treinados por 5 dias consecutivos, em seguida submetidos a ovariectomia, permanecendo em repouso por 24 horas. Exercitaram por $30 \mathrm{~min}$., 5 dias/semana durante 9 semanas com velocidade de $0,31 \mathrm{~m} / \mathrm{s}$. Foram sacrificadas e os femures pesados em balança de precisão. Os pesos encontrados: G1 $(1,68 \pm 0,15) \mathrm{g}$; G2 $(1,41 \pm 0,06) \mathrm{g}$ e $\mathrm{G} 3(1,62 \pm 0,13) \mathrm{g}$. A comparacao do G1 com G2 e G2 com C3 a diferença estatística foi significativa. G1 com G3 não apresentou diferença estatística significativa. O exercício aplicado no estudo preveniu a alteração da massa óssea provocada pela ovariectomia.

\section{Palavras-Chave}

Atividade física; Osso; Osteoporose

\section{IMPACT OF THE PHYSICAL ACTIVITY ON MASS BONY OF RATS OSTEOPENICAS}

Lorena F. Caixeta

Andre Adriano R. Aleixo

Gustavo S. Abrahão

Dayana P. P. de Siqueira

Leonardo C. Carvalho

Joao Paulo C. Matheus

Antonio C. Shimano

\begin{abstract}
We evaluated the mass of femurs of rats ovariectomized and exposed to physical activity. To perform this experiment 30 rats divided in groups: G1: Control. G2: ovariectomized animals, not submitted to exercise. G3: group the animals were trained for 5 consecutive days and then submitted to the ovariectomy, remaining in rest for 24 hours. They were submitted to physical activity for 30 min., with velocity of $0,31 \mathrm{~m} / \mathrm{s}, 5$ days/week during 9 weeks. They were sacrificed and the femurs in a heavy balance with precision. The weights found: G1 $(1.68 \pm 0.15) \mathrm{g} ; \mathrm{G} 2(1.41 \pm 0.06) \mathrm{g}$ and G3 $(1.62 \pm 0.13)$ g. The comparison of the G1 to G2 and G2 with $\mathrm{C} 3$ the difference was statistically significant. G1 to G3 with not statistical importance significant. The exercise used in the study warned the change of bone mass caused by ovariectomy.
\end{abstract}

\section{Key-Words}

Physical activity; Bone; Osteoporosis 


\section{INTRODUÇÃO}

A carga mecânica promove deformação no osso e conseqüentemente gera um estímulo para uma resposta óssea local, já postulada por Wolf em 1870. A força extema aplicada, por unidade de área do osso, pode ser classificada como compressão, tração e cisalhamento. Sob carga, estas forças aparecem de forma combinada (TUMER; BURR, 1993).

A deformação parece reduzir a reabsorção óssea e estimular a formação. Pead e Lanyon (1989) observaram elevação do número de osteoblastos formando material ósseo, na região do periósteo, em resposta à carga.

Atividades diárias geram forcas sobre os tecidos e essas forcas são ampliadas durante a pratica de esportes, como atletismo e exercícios pesados (CHARMAN, 1990). O aumento de massa óssea relacionada a atividade física e a sua diminuição ocorrida em pacientes acamados e em lesados medulares comprovam a grande influencia do estimulo biomecânico sobre o esqueleto. Portanto, o esqueleto humano e sensível aos estímulos físicos e responde a eles através de alterações tanto na massa óssea quanta em sua arquitetura (DUNCAN; TUMER, 1995). O estimulo mecânico dinâmico e mais eficaz como precursor da formação de massa óssea, pois sob cargas estáticas, as células se acomodam, tornando-se menos responsivas aos estímulos (TUMER, 1998).

Os ossos e tecidos conjuntivos quando sofrem deformação geram potenciais elétricos locais, denominados "potenciais gerados por deformação" (SGPs). Alem disso, esta deformação gera gradientes de pressão dentro dos canalículos ósseos e conseqüentemente desloca o fluxo intersticial existente neles (DUNCAN; TUMER, 1995). O aumento da deformação óssea provoca aumento do fluxo de fluido intersticial.

Estudos sugerem que a geração deste fluido circulante e importante para que o osso perceba e responda aos estímulos mecânicos (DUNCAN; TUMER, 1995). Assim, a deformação mecânica na matriz óssea e transmitida para os osteócitos, o que possibilita alterações na regulação da proliferação celular, diferenciação, morfogênese e expressão genética.

Segundo Fyhrie e Kimura (1999) o transporte de material através dos tecidos e de fundamental importância para a realização de funções e para o metabolismo homeostatico. Assim, estes autores hipotetizaram que a densidade do osso trabecular e cortical poderia estar relacionada com o transporte 
de metabolites, e que a carga mecânica elevaria o suprimento destes metabolites, da medula para a matriz óssea. Desta forma, o transporte rápido de metabolites da superfície trabecular para o interior dela ocorre pela deformação mecânica. E com este transporte dificultado, a quantidade de matriz óssea tende a diminuir para garantir a integridade de osteocitos situados no interior das trabéculas (CARVALHO, 2001).

Este estudo objetivou verificar a ação da atividade física sobre as características biomecânicas do tecido ósseo.

\section{MATERIAIS E MÉTODOS}

Foram utilizadas 30 ratas da raça Rattus norvegicus albinus da variedade Wistar, com idades entre 120 e 140 dias, apresentando peso corporal médio entre 360 e 390g. Estes animais foram divididos aleatoriamente em 3 grupos, os quais permaneceram em gaiolas de contenção. Os grupos foram caracterizados como G1: controle, com 10 animais apenas mantidos por 9 semanas em gaiolas de contenção; G2: constituído por 10 animais submetidos ao procedimento de ovariectomia; G3: onde 10 animais foram treinados em uma gaiola giratória por cinco dias consecutivos e em seguida, foram submetidos a ovariectomia, permanecendo em repouso por 24 horas. Cumprido o período de repouso os mesmos foram colocados para prática de atividade física em uma gaiola giratória por 9 semanas.

\section{PROCEDIMENTO CIRÚRGICO}

Realizou-se uma incisão reta transversal na fossa ilíaca de aproximadamente três centímetros na pele e tecido celular subcutâneo a cerca de um centímetro da linha mediana. Foi divulsionada a parede muscular ate ter acesso a cavidade abdominal, localizando-se o ovário em meio a uma massa gordurosa. A retirada do ovário foi efetuada apos ligadura da extremidade da tuba uterina, seccionando-se entre a ligadura e o ovário. A ferida foi fechada por planos. Todo o procedimento foi repetido do outro lado para a retirada do outro ovário.

\section{GAIOLA GIRATÓRIA PADRÃO}

Para execução da atividade física foi construída uma gaiola giratória em ação, fechada com tela de alumínio, com 0,96 m de perímetro e 0,12 m de largura. O modelo de gaiola e semelhante ao utilizado por Hoshi et al. (1988) e Wu et al. (2001). Um motor de corrente continua foi ligado a uma fonte possibilitando o controle da velocidade. 
Os animais exercitados tiveram suas atividades físicas divididas em duas fases, a primeira considerada treinamento e a segunda tratamento. Na fase I os animais foram submetidos a 5 dias consecutivos de treinamento com aumento progressivo do tempo de atividade física realizando 5 minutos de atividade no primeiro dia, 10 minutos no segundo, 15 minutos no terceiro, 20 minutos no quarto e 25 minutos no quinto dia sempre com velocidade media de $0,31 \mathrm{~m} / \mathrm{s}$. O treinamento foi feito antes da execução da cirurgia. Na fase II foi realizada atividade física em uma gaiola giratória por 30 minutos, 5 dias por semana durante 9 semanas com velocidade de $0,31 \mathrm{~m} / \mathrm{s}$, iniciando 24 horas apos a realização do procedimento cirúrgico.

\section{PREPARE E ANALISE DOS ESPÉCIMES}

As ratas foram mortas através da administração excessiva do anestésico Tiopental ${ }^{\circledR}$, os femures direitos e esquerdos dissecados e as peças identificadas. Em seguida a massa dos femures foi mensurada por meio de uma balança eletrônica com precisão de 0,01g. Os valores obtidos foram registrados e posteriormente submetido a analise estatística.

Os dados foram submetidos analise de variância (ANOVA), quando comparados simultaneamente. $\mathrm{Na}$ analise entre os grupos foi utilizado o teste Student -Newman - Keuls. Com niveis de significancia de 5\%. Todo método estatístico foi realizado com o programa Instat ${ }^{\circledR}$ - Graph Pad V. 3.0.

\section{RESULTADOS}

O Quadro 1 mostra os valores médios e o desvio padrão (DP) da massa dos ossos dos animais pertencentes ao grupo controle (1) que são de $(1,68 \pm 0,15) \mathrm{g}$; ovariectomizados (2) apresentando $(1,41 \pm 0,06) \mathrm{g}$ e ovariectomizados submetido a atividade física $(1,62 \pm 0,13)$. E observada diferença estatisticamente significa na massa dos ossos dos animais dos três grupos $(p=0,0189)$ comparados simultaneamente.

Se for comparado o grupo 1 versos o grupo 2 encontra - se diferença estatisticamente significativa $(\mathrm{p}<0,05)$; grupo 2 versos 3 apresenta diferença estatisticamente significativa $(\mathrm{p}<0,05)$; grupo 1 versos grupo 3 não apresenta diferença estatisticamente $(\mathrm{p}>0,05)$. 
QUADRO 1- Valores médios e desvio padrão da massa dos fêmures dos três grupos.

\begin{tabular}{ccc}
\hline Grupo & Numero & Massa dos fềmures $(\mathrm{g})$ \\
\hline Controle & 20 & $1,68 \pm 0,15$ \\
\hline Ovariectomizado & 20 & $1,41 \pm 0,06$ \\
\hline $\begin{array}{c}\text { Ovariectomia e Atividade } \\
\text { Fisica }\end{array}$ & 20 & $1,62 \pm 0,13$ \\
\hline $\mathrm{P} 00,05$ & &
\end{tabular}

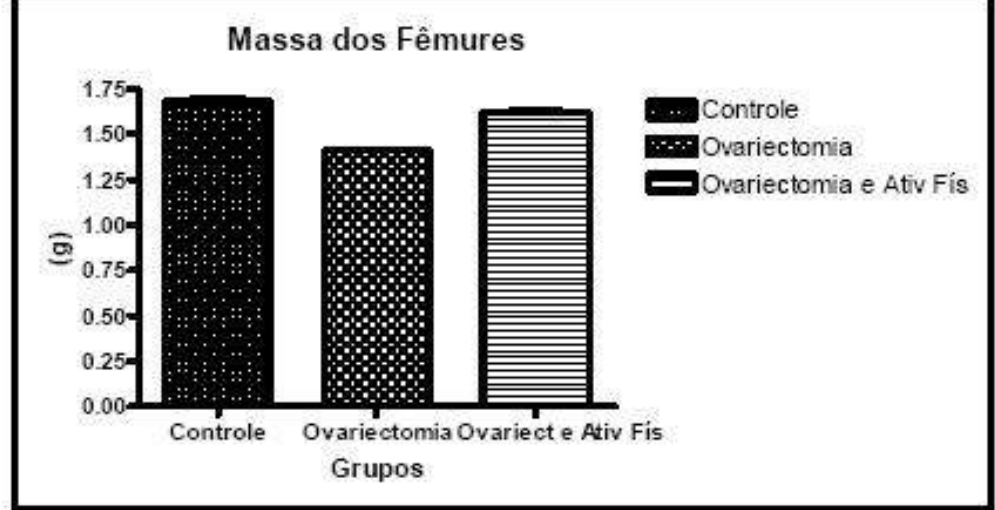

FIGURA 1- Gráfico da massa dos fêmures dos animais ovariectomizados e submetidos ao protocolo de atividade

\section{DISCUSSÃO}

O esforço físico e um estímulo importante para a renovação e o fortalecimento da estrutura óssea, porem os mecanismos pelos quais o esqueleto responde a atividade física ainda não estão totalmente elucidados. Existem, no entanto, evidencias que demonstram o aumento da resistência óssea em resposta a aplicação de cargas mecânicas e, em contrapartida, a diminuição da densidade mineral óssea (DMO), quando de sua ausência. Deve-se salientar os benefícios da atividade física em duas situações: prevenção e tratamento (RENNO et al., 2001).

Tem sido observado que atletas tem massa óssea maior do que indivíduos não atletas e a comparação entre populações ativas e sedentárias confirma uma expressiva correlação entre o nível de atividade física e a DMO. Alem disso, nota-se que a inatividade física causa osteopenia, um exemplo disso e a conhecida osteopenia decorrente da imobilização de uma fratura (KARAM, 1997). O Núcleo de Pesquisa em Atividade Física e Saúde da Universidade Federal de Santa Catarina comparou 30 mulheres sedentárias e pos - menopausicas (idade media de 61 anos) com um grupo de 20 mulheres com características semelhantes que se exercitavam moderadamente ( $75 \%$ da freqüência cardíaca máxima) no período de 2 a 4 anos. As mulheres que se exercitavam apresentaram DMO maior em todos os 
segmentos da coluna e fêmur (OURIQUES, 1997).

Em 1983; o Melpomene institute for women's health reserch (EUA) deu inicio a um estudo sobre os efeitos do estilo de vida sobre a osteoporose. Desse modo, 111 mulheres participaram da pesquisa (57 fisicamente ativas e 54 fisicamente inativas). Apos 7 anos, as mulheres foram reavaliadas e descobriu-se que 52 delas, com DMO normal, haviam praticado, nos últimos 7 anos, pelo menos 3 (sustentação de peso, aeróbico e regular) das 4 séries de exercícios. As mulheres que apresentaram DMO baixa haviam levado uma vida sedentária (BRODIGAN,1997).

No estudo encontramos resultados semelhantes aos presentes na literatura onde indivíduos ovariectomizados apresentaram valores inferiores de massa óssea do que os não ovariectomizados. Confirmando a relação direta entre a perda óssea e a falência ovariana. E que os indivíduos ovariectomizados praticantes de atividade física apresentaram valores superiores de massa óssea do que os ovariectomizados sedentários se assemelhando aos animais do grupo controle. Fornecendo indícios de que a pratica regular de exercícios possa atenuar a perda decorrente da falência ovariana.

O exercício serve de estímulo para a secreção de determinados hormônios e de fator inibitório para outros. Não se sabe o motivo das alterações nos ritmos de secreção hormonal em todas as glândulas nem nos seus níveis plasmáticos (CANALI; KRUEL, 2001). No entanto, e prudente considerar que existem motivos para essas alterações - embora ainda desconhecidos pela ciência ao invés de considerar que eles simplesmente acontecem a esmo (GOULD,1989).

E sabido que, com o exercício, a liberação do hormônio do crescimento humano ou GH (de "Human Growth Hormone") e estimulada (TSUJI et al., 1993; DEUCHLE et al., 1998).

O GH tem sua liberação controlada por um hormônio hipotalamico, o GHRH ("growth hormone release hormone") e segundo alguns autores, Berne e Levy (1996), possuem funções importantes no organismo humano, dentre elas, a estimulação do crescimento da cartilagem e do osso. Segundo MCDERMOTT (1997), a criança ativa, tem mais tendência a atingir uma altura maior do que outra sedentária.

A longo prazo, o exercício causa a formação óssea (CANALI; KRUEL, 2001). Isso e resultado primariamente da absorção intestinal aumentada de cálcio, junto com a diminuição de sua excreção pela urina e com níveis aumentados do hormônio paratireoidiano (PTH). Ao contrario, a imobilização ou repouso completo promove diminuição óssea (CANALI e KRUEL, 2001). 
Um dos principais requisites para a formação óssea e a forca da gravidade. Foi verificado nos astronautas, quando de seu retorno a Terra, que estes apresentavam uma perda de massa óssea significativa, apesar de mantidos os exercícios durante as viagens espaciais (PLAPLER, 1997).

A resposta mecânica de qualquer material ou estrutura a uma forca aplicada e definida em termos de tensão (stress) e deformação (strain). Tensão e a medida de forca aplicada em uma certa área. Quando uma forca e aplicada em um osso, seu comprimento se modifica. Esta mudança no comprimento e definida como deformação (DUNCAN; TUMER, 1995).

Com relação a carga, alguns estudiosos, como Galilleo em 1683, reconhece a correlação entre o peso corporal e o tamanho dos ossos, mas foi Wolff em 1892, quem observou que mudanças na massa óssea acompanham mudanças de carga, levando a remodelação óssea (KARAM, 1997).

A piezoeletricidade do osso foi observada em 1957 por Fukuda. O efeito piezoeletrico e aquele verificado em um material, quando este produz, sob carga mecânica, uma polarização elétrica, convertendo, portanto, a energia mecânica em energia elétrica (KODAMA, 2003).

Assim, ao se aplicar uma carga mecânica no osso, ocorre a deformação nos componentes orgânicos do material, que produzem potenciais elétricos (KAPLAN et al, 1994). Os potenciais elétricos atuam como um sistema sinalizador para os osteoblastos e osteoclastos, fazendo com que ocorra o aumento/diminuição da formação de tecido, ou o aumento/diminuição da absorção de tecido, como uma resposta ao estimulo mecânico (CHARMAN, 1990).

As forcas de compressão são realizadas por atividades físicas de impacto e cargas exercidas pelos tendões e músculos sobre os ossos. Dai a importância da atividade física para a manutenção da integridade do tecido ósseo (RENNO et al, 2001).

Um outro ponto importante para compreensão dos efeitos da atividade física sobre a remodelação óssea e a duração do estimulo a ser aplicado. A grande maioria das evidencias indica que a duração do estimulo não é o dado mais relevante. Esses achados corroboram com teorias que afirmam que o tecido ósseo só responde acima ou abaixo de um limiar definido (RENNO et al., 2001).

Chen et al. (2004) avaliaram a influencia do exercício na densidade mineral óssea e na propriedade mecânica de rigidez. Os fêmures de ratos exercitados por 4 semanas apresentaram valores superiores ao 
grupo não exercitado.

No entanto, o estudo de Abrahao et al. (2006), demonstraram que a pratica de atividade física associado a ovariectomia, não foi suficiente para corrigir completamente a perda de resistência mecânica, mas propiciou maior suporte de carga.

Segundo Yoshimura (2003); Eastell (2003); Singh (2004) a atividade física e um importante recurso para a manutenção da qualidade óssea. Sendo capaz de prevenir a perda óssea secundaria, a deficiência estrogênica e reduzir significativamente o risco da ocorrência de fraturas em pacientes com osteoporose.

Licy, et al. (2003) realizaram um estudo avaliando a ação da atividade física e reposição de estrogênio em ratas ovariectomizadas na prevenção de perda óssea do colo femoral e vértebras lombares. Os resultados histomorfometricos evidenciaram que a associação de atividade física e reposição hormonal promoveram a elevação da massa óssea das regiões estudas.

Going et al. (2003), realizaram um estudo com 320 mulheres no período pos menopausa para avaliar a combinação de exercícios, terapia de reposição hormonal e administração de cálcio. Apos 12 meses foi constatado que as mulheres submetidas a atividade física e reposição hormonal apresentaram os melhores resultados de densidade óssea.

Segundo Hoshi et al. (1998), o exercício reduz a perda de massa óssea associada com a idade. Wu et al. (2001) afirmaram que a pratica de exercícios moderados e a administração de doses reduzidas de hormônios apresentam um efeito cooperativo na prevenção de perda óssea em ratas ovariectomizadas. A modalidade, intensidade e freqüência de exercícios indicadas para pacientes com osteoporose ainda são controversas. Desta maneira, e fundamental que se desenvolvam pesquisas com a finalidade de estabelecer parâmetros adequados (RENNO,2001).

\section{CONCLUSÃO}

O exercício aplicado por $30 \mathrm{~min}, 5$ dias por semana durante 9 semanas a $0,31 \mathrm{~m} / \mathrm{s}$ foi capaz de prevenir a alteração da massa ósseo provocada pela ovariectomia.

Sugerindo que a pratica de atividade física regular e um importante estimulo para manutenção das propriedades biomecânicas e homeosetasia mineral do esqueleto. 


\section{REFERÊNCIAS}

ABRAHÃO, G. S.; SHIMANO, A. C.; PICADO, C. H. F. Ação da atividade física sobre as propriedades mecânicas dos fêmures e tíbias de ratas asteopenicas. Acta Ortopédica Brasileira, São Paulo, v. 14, n. 5, p.242-245, 2006.

BERNE, R. M.; LEVY, M. N. Fisiologia. 3.ed. Rio de Janeiro: Guanabara Koogan, 1996.

BRODIGAN, D. E. Osteoporose: efeito devido a exercícios físicos. Revista de Oxidologia, Sao Paulo, p.28-34, nov./dez. 1997.

CANALI, E. S.; KRUEL, L. F. M. Respostas hormonais ao exercício. Revista Paulista de Educação Física, São Paulo, v.15, n.2, p.141-53, jul./dez. 2001.

CARVALHO, D. C. L. Ação do ultra-som de baixa intensidade em ossos de ratas osteopênicas. 2001. 82f. Dissertação (Mestrado) - Faculdade de Medicina de Ribeirão Preto, Universidade de São Paulo, São Carlos, 2001.

CHARMAN, R. A. Strain generated potencials in bone strength, bone mineral density, end metal content in rat femurs. Bio-medical Materials and Engineering, Amsterdam, v. 14, n. 1, p. 53-59, 1990. CHEN, X.; AOKI, H.; FUKUI, Y. Effect of exercise on the bone strength, bone mineral density, and metal content in rat femurs. Bio-medical Materials and Engineering, Amsterdam, v. 14, n. 1, p. 53-59, 2004.

DEUSCHLE, M. et al. Endurance training and its effect upon the activity of the GH-IGFs system in the elderly. Intemational Joumal of Sports Medicine, Stuttgard, v.19, p.250-253, 1998.

DUCAN, R. L.; TUMER, C. H. Mechanotransduction and the functional response of bone to mechanical strain. Calcified Tissue Intemational, New York, v.57, p.344-358, 1995.

EASTELL, R. Management of osteoporosis due to ovarian faiture. Medical an Pediatric Oncology, New York, v. 41, n. 3, p. 222-227, 2003.

GOING, S. et al. Effects of exercise on boné mineral density in calcium-replete postmenopausal women with and without hormone replacement therapy. Osteoporosis Intemational, London, v. 14, n.8, p.637643, 2003.

GOULD, J. A. (1989). O polegar do panda: reflexões sobre história natural. São Paulo: Martins Fontes, 1989.

HOSHI, A. et al. Effects of exercise at different ages on bone density and mechanical properties of femoral bone of aged mice. Tohoku Joumal Exp Medical, Tokyo, v. 5, p. 15-24, 1998.

KAPLAN, F. S. et al. (1994). Form and function of bone. In: SIMON, S.R.; WILSON, J. Orthopaedic basic science. American Academy of Orthopaedic Surgeons. Cap. 4, p.127-184. 
KARAM, F. C. Esporte como prevenção de osteoporose: um estudo da massa óssea de mulheres pósmenopáusicas que foram atletas de voleibol.Porto Alegre, 102f. Dissertação (Mestrado) - Universidade Federal do Rio Grande do Sul, Porto Alegre, 1997.

KODAMA, A. C. Efeitos do ultra-som pulsado de baixa intensidade em um modelo osseo de ratas ovarectomizadas analisadas por ensaios de flexo-compressão. São Carlos. Dissertação (Mestrado) Faculdade de Medicina de Ribeirao Preto, Universidade de São Paulo, São Carlos. 2003.

LICY, J. W. S. et al. Estrogen and "exercise" have a synergistic effect in preventing bone loss in the lumbar vertebra and femoral neck of the ovariectomized rat. Calcified Tissue Intemational, New York, v. 72, n. 1, p. 42-49, 2003.

MCDERMOTT, M. T. Segredos em endocrinologia. Porto Alegre: Artes Medicas, 1997.

OURIQUES, E. P. M. Atividade fisica; uma forma natural de prevenir osteoporose. In: CONGRESSO PANAMERICANO DE MEDICINA DO ESPORTE, 17./congresso brasileiro de medicina do esporte, 12., 1997, Gramado. Anais... Gramado: Confederação Pan-americana/SBME, 1997 p. 49.

PLAPLER, R. G. Osteoporose e exercícios. Revista do Hospital das Clinicas da Faculdade de Medicina, São Paulo, 1997; v. 52, n. 3, p.163-170, 1997.

RENNO, A. C. M.; DRIUSSO, P.; FERREIRA, V. Atividade física e osteoporose: uma revisão bibliográfica. Fisioterapia em Movimento, Curitiba, v.13, n.2, p.49-54, mar. 2001.

SINGH, M. A. Physician activity and boné heath. Australian Family Physician, South Melboume, v. 33, n. 3, p. 125-149, 2004.

TSUJ, H.; CURI, P. R.; BURINI, R. C. Alterações metabólicas e hormonais em nadadores durante o treinamento físico. Revista Brasileira de Ciências e Movimento, São Caetano do Sul, v.7, n.2, p.35-41, 1993.

TUMER, C. H.; BURR, D. B. Basic biomechanical measurements of bone: a tutorial. Bone, Elmsford, v.14, n.4, p.599-608, jul./ago. 1993.

WU, J. et al. Cooperative effects of exercise training and genistein admistration on bone mass in ovarectomized mice. Joumal of Bone and Mineral Research, New York, v. 16, n. 10, p.1829-1836, oct. 2001.

YOSHIMURA, N. Exercise and physical activites for the prevention of osteoporótico fractures: a review of the evidence. Nippon Eiseigaku Zasshi, Tokyo, v. 58, n. 3, p. 328-337, 2003. 


\section{Lorena Ferreira Caixeta}

Universidade de Uberaba

\section{Andre Adriano R. Aleixo}

Universidade de Uberaba

\section{Gustavo S. Abrahão \\ FMRP-USP}

\section{Dayana P. P. de Siqueira \\ USP}

\section{Leonardo C. Carvalho \\ FMRP - USP}

\section{Joao Paulo C. Matheus}

FMRP - USP

\section{Antonio C. Shimano}

FMRP-USP.

\section{Referência do artigo:}

\section{ABNT}

CAIXETA, L. F. al. Impacto da atividade física sobre a massa óssea de ratas osteopenicas. Conexões, v. 6, p. 261-271, 2008.

APA

Caixeta, L. F., Aleixo, A. A. R., Abrahão, G. S., Siqueira, D. P. P., Carvalho, L. C., Matheus, J. P. C., \& Shimano, A. C. (2008). Impacto da atividade física sobre a massa óssea de ratas osteopenicas. Conexões, 6, 261-271.

\section{VANCOUVER}

Caixeta LF, Aleixo AAR, Abrahão GS, Siqueira DPP, Carvalho LC, Matheus JPC, Shimano AC. Impacto da atividade física sobre a massa óssea de ratas osteopenicas. Conexões, 2008; 6: 261-271. 